\title{
Transcriptome analysis reveals different responses and tolerance mechanisms of EPSPS and GAT genes in transgenic soybeans
}

\section{Bingfu Guo ( $\sim$ gbfhq@163.com )}

CAAS ICS: Chinese Academy of Agricultural Sciences Institute of Crop Sciences

Hui-Long Hong

CAAS ICS: Chinese Academy of Agricultural Sciences Institute of Crop Sciences

\section{Yong Guo}

CAAS ICS: Chinese Academy of Agricultural Sciences Institute of Crop Sciences

\section{Li-Juan Qiu}

CAAS ICS: Chinese Academy of Agricultural Sciences Institute of Crop Sciences

\section{Research Article}

Keywords: Soybean, RNA-seq, Glyphosate tolerance, EPSPS, GAT

Posted Date: May 26th, 2021

DOI: https://doi.org/10.21203/rs.3.rs-176579/v1

License: (a) (i) This work is licensed under a Creative Commons Attribution 4.0 International License. Read Full License 


\section{Abstract}

Background: Glyphosate is a broad-spectrum and non-selective systemic herbicide. Introduction of glyphosate tolerance genes like EPSPS or detoxification genes like GAT could confer glyphosate tolerance to plants. Our previous study revealed that co-expression of EPSPS and GAT genes conferred higher glyphosate tolerance with no "yellow flashing" phenomenon. However, the tolerance mechanisms response to glyphosate at transcriptional level was still unknown.

Methods and Results: To investigate glyphosate tolerance mechanisms in different GM soybeans, RNAseq analysis was conducted using four soybean genotypes, including two non-transgenic (NT) soybeans ZH10, MD12, and two GM soybeans HJ698 and ZH10-6. A total of $90.72 \mathrm{~Gb}$ clean reads were generated and differentially expressed genes (DEGs) were identified in these soybeans before and after glyphosate treatments. The similar response of glyphosate in NT soybeans and the different effect of glyphosate to two GM soybeans were identified. The number of DEGs involved in shikimate biosynthetic pathway and herbicide targeted cross-pathways was continuously increased in NT soybeans, and slightly increased in HJ698 as the increasing of treat time. However, it was only significantly increased at $12 \mathrm{hpt}$ but tended to recover to levels of $0 \mathrm{hpt}$ at $72 \mathrm{hpt} \mathrm{in} \mathrm{ZH} 10-6$, which can explain the higher glyphosate tolerance of $\mathrm{ZH} 10-$ 6.

Conclusions: All these results suggested that GAT and EPSPS genes associated play a crucial role in response to glyphosate, and the former might work at the early stage of glyphosate exposure while the latter might be activated after the uptake of glyphosate uptake. These findings will provide valuable sights for understanding of the molecular basis underlying glyphosate tolerance.

\section{Introduction}

Soybean (Glycine max L.) is the most frequently cultivated grain legume crops all over the world [1]. With the development of transgenic technology, commercially available genetically modified (GM) soybeans are mostly tolerant to glyphosate or glufosinate. Glyphosate is the most widely used broad-spectrum, non-selective herbicides. The plant endogenous 5-enolypyruvylshikimate-3-phosphate synthase (EPSPS) activity is highly inhibited by glyphosate, leading to the accumulation of shikimate and the deficiency of chorismate in the shikimate pathway [2]. However, the chorismate is the indispensable precursor for biosynthesis of aromatic amino acids in plants, including phenylalanine, tyrosine and tryptophan $[2,3]$. To date, majority of glyphosate-tolerant GM soybeans are derived from soybean event GTS 40-3-2, which contains one functional insert expressing CP4-EPSPS gene (http://www.isaaa.org). CP4-EPSPS encodes 5-enolypyruvylshikimate-3-phosphate synthase (EPSPS) whose low affinity for glyphosate and high catalytic activity contributes to glyphosate tolerance in plants [4].

Some studies reported that overexpression of exogenous EPSPS conferred glyphosate tolerance in plants, but the applied glyphosate was found to accumulate in non-transgenic (NT) and GM plants [5-8]. In turn, its accumulation led to the decrease of crop yield by interfering with photosynthesis, chlorophyll content, 
shikimate content and nutrient accumulation [9]. The glyphosate tolerance does not mean the reducing of glyphosate translocation or accumulation in GM plants, and the main concern is the toxic injury caused by glyphosate accumulation. Previous studies also showed that the glyphosate accumulation led to the decrease of chlorophyll content, accumulation of primary phytotoxic metabolite or the formation of insoluble glyphosate-metal complexes [9-11]. Interestingly, the typical symptom of glyphosate application, which is known as "yellow flashing", was observed in not only NT soybean but also GM soybean only expressed with EPSPS gene [9]. With the identification of more glyphosate-related genes, the glyphosate $\mathrm{N}$-acetyltransferase gene (GAT) or glyphosate oxidoreductase gene (GOX) was used in detoxification of glyphosate [12-14]. Co-expression of GAT and EPSPS genes conferred higher tolerance to glyphosate in tobacco, cotton and soybean, and the "yellow flashing" symptom was not found after glyphosate application $[8,15,16]$. Moreover, the significant changes of shikimate acid content and chlorophyll content were not detected in GM soybean expressed with EPSPS and GAT genes, suggesting that the shikimate pathway could not be inhibited [16].

Over the past years, some reports have focused on the glyphosate-response mechanism and gene expression profiling pattern with glyphosate treatment in NT soybean and GM soybeans expressed with CP4-EPSPS $[17,18]$. However, little is known about how plants response to glyphosate when glyphosate exposure, particularly the transcriptional changes in plants expressed glyphosate tolerance EPSPS and detoxification gene GAT. In this study, two NT soybeans (ZH10 and MD12) and two GM glyphosatetolerant soybeans (ZH10-6 and HJ698) were selected for transcriptome analysis. Transcriptional changes of these soybean genotypes contrasting in their tolerance to glyphosate at three time points were revealed by RNA-seq and thousands of differentially expressed genes were identified. The objective of this study was to investigate the gene expression profiles of these soybeans, and to explore the possible mechanism in response to glyphosate. The results will provide not only a basis for understanding the mechanism in response to glyphosate, but also valuable clues for further glyphosate research in crops.

\section{Materials And Methods}

\section{Plant materials and growth conditions}

Glyphosate-tolerant soybeans ZH10-6, HJ698 and NT soybean cultivars Zhonghuang10 (ZH10), Mengdou12 (MD12) were used for glyphosate treatment and transcriptome sequencing. Among them, ZH10-6 expressed with glyphosate tolerance gene G2-EPSPS and glyphosate-degrading gene GAT was developed by Agrobacterium-mediated transformation of ZH10 [16]. HJ698 is a backcross line derived from MD12 and GTS40-3-2. Soybean seeds were planted in $10 \mathrm{~cm}$ plastic pots containing mixture of vermiculite, peat, nutrient soil with the ratio of 1:2:4 and grown in the controlled chamber under a $16 \mathrm{~h} / 8 \mathrm{~h}$ photoperiod with $120 \mu \mathrm{mol} / \mathrm{m}^{2}$.s light intensity. Day and light temperatures were $26^{\circ} \mathrm{C}$ and $24^{\circ} \mathrm{C}$, respectively. Three plants per pot were reserved after seedling emergence.

\section{Glyphosate treatments}


Commercially formulated isopropylamine salt of glyphosate with the rate of $300 \mathrm{~g}$ a.e./L (RoundupÒ, Monsanto Company) was used for glyphosate treatment. The labeled rate for glyphosate application varies from 600 to $1200 \mathrm{~g}$ a.e. /ha [39]. When the first trifoliolate leaves of seedlings were fully expanded, soybean plants were spraying with Roundupò at the rate of $0.18 \mathrm{~g}$ a.e. $/ \mathrm{m}^{2}$ as reported previously [16], which is a relatively high dosage represent the "worst-case scenario" to promote injury. The phenotype of whole seedlings were observed before ( $0 \mathrm{hpt})$ and $12,24,72$, and 120 hours post treatment $(12,24,72$ and $120 \mathrm{hpt}$ ). At the same time, leaves from ten plants of these four soybean genotypes were collected at Ohpt, $12 \mathrm{hph}$ and $72 \mathrm{hpt}$. All samples were immediately frozen in liquid nitrogen and stored at $-80^{\circ} \mathrm{C}$ until RNA extraction.

\section{RNA extraction and sequencing}

Total RNA was extracted from leaves with RNA extraction kit (TRIzol reagent, Invitrogen Inc) following the manufacturer's instructions. The quality and quantity of RNA were assessed with agarose gels electrophoresis and a Nanodrop spectrophotometer (Thermo Scientific, USA). A total of 14 libraries were constructed using reagents available in the RNA-Seq Sample Preparation Kit (Illumina, USA). All these libraries were sequenced and paired-end reads were generated using the Illumina HiSeq 2500 platform.

\section{RNA-Seq data filtering and analysis}

Quality control of raw reads was performed using BMKCloud software (https://www.biocloud.net/) and high quality clean reads were obtained through removal of adaptor sequences, ambiguous reads with ' $N$ ' bases and low quality reads in which $>10 \%$ of bases had a Q-value less than 20 . Then all clean reads were aligned to the soybean reference genome of $\mathrm{cv}$. Williams 82 (https://phytozome.jgi.doe.gov/pz/portal.html\#!info?alias=Org_Gmax) using TopHat software (http://ccb.jhu.edu/software/tophat/index.shtml). After passing the process of the alignment, the duplicate reads were removed and the remaining reads were used for further analysis.

\section{Differential gene expression analysis}

Gene expression analysis was performed by Cufflink 2.2.1 (http://cole-trapnelllab.github.io/cufflinks/releases/v2.2.1/). Fragments per Kilobase of transcript per Million fragments mapped (FPKM) [19] was used to normalize the gene transcript levels. Transcript abundance of all genes in different samples was estimated by calculating the read density. DEGs were identified among fourteen libraries using EBSeq software [20]. A threshold for false discovery rate (FDR) $<0.01$ and an absolute value of log2 fold-change $(F C) \geq 1$ were used to identify significant expression changes.

\section{qRT-PCR analysis}

In order to verify the expression abundance obtained by transcriptome sequencing, 15 genes were selected for qRT-PCR analysis in different samples. All the primers used for qRT-PCR were listed in Supplementary Table S1. cDNA synthesis and qRT-PCR analysis were performed as previously described 
[16]. The relative expression level was calculated using $2^{-\Delta \Delta t}$ method and standardized to the constitutive expression level of Actin [21]. Three biological replicates were used for each gene.

\section{Functional annotation}

To acquire the most comprehensive annotation, functional annotations were performed by sequences similarity search against five public databases. All genes were aligned with NCBI non-redundant protein database (Nr) [22], Gene ontology (GO) [23], Clusters of Orthologous Groups (COG) [24], SWISS-PROT [25] and the Kyoto Encyclopedia of Genes and Genomes databases (KEGG) [26]. Blast2GO was used to obtain GO functional categories [27].

\section{Selection of genes involved in specific pathways and heatmap analysis}

Glyphosate is proved to specifically inhibit the synthesis of phenylalanine, tyrosine and tryptophan in the shikimate pathway, which serve as the precursors of numerous compounds such as pigments, alkaloids, hormones and cell wall components [40]. Based on the annotation information in NCBI database, DEGs involved in some specific pathways were selected for detailed analysis, and Heatmap analysis of DEGs was performed by BMKCloud (https://www.biocloud.net/).

\section{Results}

\section{Phenotypic and glyphosate tolerance analysis}

To determine the different tolerance to glyphosate, seedlings of ZH10, MD12, ZH10-6 and HJ698 were treated with Roundupò at the dose of $0.18 \mathrm{~g}$ a.e. $/ \mathrm{m}^{2}$. There was no detectable growth difference at 12 hpt in these four genotypes after treatment. As the observation time increased $(24,72,120 \mathrm{hpt})$, NT soybeans $\mathrm{ZH} 10$ and MD12 had visible foliar damage such as leaf roll, leaf chlorosis, and even leaf dehydration, whereas only leaf chlorosis could be observed in GM soybean HJ698 at 120 hpt but not in GM soybean $\mathrm{ZH} 10-6$. These results indicated that $\mathrm{ZH} 10-6$ and HJ698 showed tolerance to glyphosate and $\mathrm{ZH} 10-6$ displayed better growth condition after glyphosate treatment (Fig. 1). Interestingly, the "yellow flashing" phenomenon was only observed in terminal leaflets of HJ698 (Fig. 1). In other words, overexpression of EPSPS contributed to improve glyphosate tolerance in soybean, while overexpression of EPSPS and GAT conferred higher tolerance to glyphosate.

\section{RNA-seq analysis}

To compare the whole transcriptome response to glyphosate between these soybean genotypes, RNA samples were isolated from terminal leaflets after glyphosate treatment $(0,12$ and $72 \mathrm{hpt})$, respectively. 14 cDNA libraries were constructed for RNA-seq with two biological replications each for two time points (ZH10-6_12 hpt and HJ698_12 hpt). A total of 388 million raw reads were obtained and $90.72 \mathrm{~Gb}$ sequencing data was generated, with at least $4.8 \mathrm{~Gb}$ for each sample on average after data filtering. The average ratio of clean reads and GC content were about $91.5 \%$ and $49.8 \%$, respectively. More than $92 \%$ of 
the reads had Phred-like quality scores at Q30 level (Supplementary Table. S2), exhibiting that the sequencing data was high quality. To detect the distribution of generated sequence, all clean reads from each library were mapped to reference genome (Supplementary Fig. S1). After genome mapping with TopHat [28], 30.5 to 57.9 million clean reads was successfully mapped to the reference genome, and the average mapping ratio was about $72.7 \%$ (Supplementary Table. S2). Pearson's correlation coefficient $\left(r^{2}\right)$ is an important index to reflect the relationships among the two biological replicates [29], and the value of $r^{2}$ of two replicated libraries was 0.9928 and 0.9836 in ZH10-6_12h and HJ698_12h, respectively, indicating that the RNA-seq data were reliable and reproducible.

\section{Identification of DEGs}

FPKM value of each gene was calculated for evaluating gene expression levels and all DEGs were identified (Supplementary Table. S3). To verify the reliability of RNA-Seq, 15 DEGs were randomly selected for qPCR assays. There was the same trend with less discrepancy between qPCR and RNA-seq results, indicating that the transcriptome data are reliable (Supplementary Table. S4). A total of 2,946 DEGs (1,365 up-regulated and 1,581 down-regulated genes) and 2,896 DEGs (1,119 up-regulated and 1,777 down-regulated genes) were identified in ZH10 and MD12 at $12 \mathrm{hpt}$, respectively (Fig. 3). In addition, 6,972 DEGs (3175 up-regulated and 3797 down-regulated genes) and 4,423 DEGs (1959 upregulated and 2464 down-regulated genes) in $\mathrm{ZH} 10$ and MD12 at $72 \mathrm{hpt} /$ respectively (Fig. 3). 4,711 DEGs (1893 up-regulated and 2818 down-regulated genes) and 4,953 DEGs (2833 up-regulated and 2120 downregulated genes) were identified in HJ698 only expressed EPSPS at 12 and $72 \mathrm{hpt}$, respectively. 7,820 DEGs (3319 up-regulated and 4501 down-regulated genes) and 2,289 DEGs (1103 up-regulated and 1186 down-regulated genes) were identified in ZH10-6 at 12 and 72 hpt, respectively (Fig. 3). Compared to 12 hpt, the numbers of DEGs slightly increased in HJ698 at $72 \mathrm{hpt}$ while the numbers of DEGs significantly reduced in $\mathrm{ZH} 10-6$ at $72 \mathrm{hpt}$.

\section{Similar response to glyphosate in two NT soybeans}

Both GO and KEGG enrichment analysis were used for identification of key genes and pathways in response to glyphosate. GO enrichment analysis showed that a large number of DEGs were enriched in response to chitin (225 genes), response to wounding (195 genes), response to water deprivation (193 genes), and defense response to fungus (191 genes) terms in ZH10_12 hpt (Supplementary Fig. S2a), while a large number of DEGs were enriched in oxidation-reduction process (537 genes), chloroplast stroma (388 genes), chloroplast envelope (368 genes), chloroplast thylakoid membrane (292 genes) and chlorophyll biosynthetic process (115 genes) terms in ZH10_72 hpt (Supplementary Fig. S2b). Similar to ZH10, DEGs were mainly associated with plant stress and defense responses in MD12_12 hpt, while associated with photosynthesis and chlorophyll biosynthesis pathways in MD12_72 hpt (Supplementary Fig. S2c and S2d).

KEGG analysis revealed that DEGs of ZH10_12 hpt were significantly grouped into plant hormone signal transduction, phenylpropanoid biosynthesis and plant-pathogen interaction categories. Among them, 
plant hormone signal transduction and plant-pathogen interaction pathways were associated with plant stress and defense response (Supplementary Fig. S4a). However, a different clustering tendency was identified at ZH10_72 hpt. DEGs grouped into 20 clusters and mainly contained porphyrin and chlorophyll metabolism, carotenoid biosynthesis, pentose phosphate pathway, glycolysis/gluconeogenesis, carbon fixation in photosynthetic organisms, photosynthesis-antenna proteins, photosynthesis pathways (Supplementary Fig. S4b). These pathways were mainly related to photosynthesis, chlorophyll metabolism, and some pathways such as pentose phosphate pathway, glycolysis and phenylpropanoid biosynthesis were located in the upstream or downstream of shikimate acid pathway. Similarly, KEGG analysis revealed that DEGs of MD12_12 hpt were significantly associated with plant stress response and defense response pathways, and DEGs of MD12_72 hpt were mainly enriched in chlorophyll biosynthesis, photosynthesis, and shikimate-related pathways (Supplementary Fig. S4c and S4d). Moreover, a few DEGs of MD12_12 hpt were found to involve in photosynthesis and carotenoid biosynthesis pathways (Supplementary Fig. S4c).

\section{Different response to glyphosate in two GM soybeans}

GO enrichment analysis revealed that DEGs of HJ698_12 hpt were classified into 20 clusters and mainly involved in plant stress response and plant defense response pathways, including oxidation-reduction process (323 genes), response to water deprivation (269 genes), response to chitin (258 genes), response to wounding (257 genes), and defense response to fungus (257 genes) (Supplementary Fig. S3a). GO enrichment analysis revealed that DEGs of HJ698_72 hpt were also mainly grouped into plant stress response and defense response pathways, and only a few DEGs were involved in chlorophyll photosynthesis and shikimate-related pathway (Supplementary Fig. S3b). Unlike two NT soybeans and HJ698, a significant difference was found in ZH10-6_12 hpt. GO enrichment analysis revealed that DEGs of ZH10-6_12 hpt were mainly involved in function of chlorophyll and photosynthesis pathways, including chloroplast stroma (369 genes), chloroplast envelope (340 genes), and chloroplast thylakoid membrane (206 genes) (Supplementary Fig. S3c). Additionally, DEGs of ZH10-6_72 hpt were mainly enriched into plants response to stress and defense pathways, which contained response to chitin (142 genes), response to water deprivation (138 genes), response to wounding (136 genes), defense response (133 genes), response to cold (128 genes) (Supplementary Fig. S3d).

KEGG analysis revealed that DEGs were clustered into similar function categories both in HJ698 at $12 \mathrm{hpt}$ and $72 \mathrm{hpt}$. These genes were mainly involved in carbon metabolism, biosynthesis of amino acids, plantpathogen interaction, flavonoid biosynthesis, phenylpropanoid biosynthesis, pentose phosphate pathway, glycolysis/gluconeogenesis, and photosynthesis (Supplementary Fig. S5a and 5b). KEGG analysis revealed that DEGs of ZH10-6_12 hpt were mainly involved in photosynthesis and energy metabolism pathway, including carbon metabolism, carbon fixation in photosynthesis organisms, photosynthesis,

TCA cycle, glycolysis/gluconeogenesis, and pentose phosphate pathway (Supplementary Fig. S5c), while DEGs of ZH10-6_72 hpt were significantly associated with plant hormone signal transduction, phenylalanine metabolism, circadian rhythm-plant, fatty acid elongation and flavonoid biosynthesis, but 
chlorophyll biosynthesis, photosynthesis and shikimate pathway was not found in ZH10-6_72 hpt (Supplementary Fig. S5d).

\section{DEGs involved in shikimate acid biosynthetic pathway}

Since glyphosate is proved to interfere with the shikimate acid biosynthetic pathway, genes involved in this pathway were selected for further analysise. 18 and 8 DEGs involved in shikimate acid pathway were identified at $12 \mathrm{hpt}$ in $\mathrm{ZH} 10$ and MD12 respectively, but this number was obviously increased into 33 and 18 at $72 \mathrm{hpt}$. Compared to NT soybeans, a relative gentle increasing trend of DEGs was observed in HJ698 (20 and 22 DEGs were identified at 12 and 72 hpt, respectively). However, a completely opposite change trend of DEGs was observed in $\mathrm{ZH} 10-6$, in which 32 DEGs involved in shikimate acid pathway were identified at $12 \mathrm{hpt}$ while only 6 were identified at $72 \mathrm{hpt}$. Meanwhile, to compare the changes of shikimate acid biosynthetic pathway among these soybeans, a heat map was generated to present the transcript abundance for all DEGs. Heatmap culster analysis revealed that expression level of many genes were continuously increased or decreased in NT soybeans and slightly altered in HJ698 as the extension of treat time. As to genes in $\mathrm{ZH} 10-6$, the trend of gene expression was totally different. The expression level of these genes was only significantly altered at $12 \mathrm{hpt}$, but it tended to recover to levels of $0 \mathrm{hpt}$ at $72 \mathrm{hpt} \mathrm{(Fig.} \mathrm{4).} \mathrm{These} \mathrm{results} \mathrm{indicated} \mathrm{that} \mathrm{shikimate} \mathrm{acid} \mathrm{biosynthetic} \mathrm{pathway} \mathrm{was}$ associated with different phenotypic variation among these soybeans.

\section{DEGs involved in herbicide targeted cross-pathways}

Interestingly, inhibition of shikimate pathway by glyphosate can specifically influence the expression of genes not only in target pathway but also cross-pathways, and the action modes of herbicides mainly include photosystem, chlorophyll biosynthesis, synthetic auxin, microtubule and cellulose biosynthesis pathways [18]. Significantly, the "yellow flashing" phenomenon was also observed in glyphosate-tolerant soybean HJ698 after glyphosate application (Fig. 1)., Previous study revealed that the decrease of chlorophyll content is the main reason for this symptom [16]. Detailed analysis of genes related to photosystem and chlorophyll biosynthesis pathways showed that the expression level of many DEGs at $72 \mathrm{hpt}$ was obviously altered than that at $12 \mathrm{hpt}$ in NT soybeans, and slightly altered in HJ698. However, the expression level of these genes was significantly altered at $12 \mathrm{hpt}$ but it tended to recover to levels of 0 hpt at 72 hpt in ZH10-6(Fig. 5).

DEGs involved in synthetic auxin, microtubule and cellulose biosynthesis pathways. A total of 117 DEGs related to synthetic auxin and $43 \mathrm{DEGs}$ involved in microtubule and cellulose biosynthesis response to glyphosate in four soybean genotypes were identified. The number of DEGs related to synthetic auxin, microtubule and cellulose biosynthesis at $72 \mathrm{hpt}$ was obviously increased in HJ698 and NT soybeans compared to $12 \mathrm{hpt}$. However a reverse change trends of No. of DEGs related to synthetic auxin and microtubule and cellulose biosynthesis was observed in ZH10-6, 60 and 21 DEGs were identified at $12 \mathrm{hpt}$ while only 12 and 6 DEGs were found at $72 \mathrm{hpt}$. Interestingly, the variation tendency of expression abundances of DEGs was obviously strengthened in NT soybeans and HJ698 as the increasing of treat time. But the abundance level at $72 \mathrm{hpt}$ was inclined to return to the level at $0 \mathrm{hpt}$ (Supplementary Fig. S6, 
S7 and S8). This result was consistent with the analysis of expression abundance of DEGs related to shikimate pathway, photosystem and chlorophyll biosynthesis pathways. These results showed that photosystem, chlorophyll biosynthesis, synthetic auxin, microtubule and cellulose biosynthesis were also associated with phenotypic variation among these soybeans after glyphosate application.

\section{Discussion}

In this study, the transcriptome analysis of four soybean genotypes in response to glyphosate were revealed by RNA-seq, and these transcriptional changes will contribute to the comprehensive understanding of DEGs or pathways involved in response to glyphosate. Similar to pathogen attacks, plant defense and stress response mechanism will be activated when glyphosate exposure, and oxidation-reduction process was indispensable defense strategy. GO enrichment analysis showed that DEGs involved in plants stress response and plant defense were mainly in NT soybeans at $12 \mathrm{hpt}$, in HJ698 at 12 and 72 hpt, and in ZH10-6 at 12 hpt (Supplementary Fig. S2 and S3). Surprisingly, DEGs associated with plant stress response and plant defense were not enriched in ZH10-6 at $12 \mathrm{hpt}$ (Supplementary Fig S3c).

Due to the non-selective characteristic of glyphosate, it is easily accumulated in plants through glyphosate spray. The uptake of glyphosate is a biphasic process which contains glyphosate penetration through the cuticle and glyphosate translocation into the symplast with phosphate transporters [30, 31]. Excessive glyphosate accumulation led to increased biological damage effects, which can be detected in NT soybeans and HJ698 (Fig 1). Visible foliar damages such as"yellow flashing" phenomenon, leaf roll and even leaf dehydration were also observed in two NT soybeans ZH10 and MD12 (Fig 1). Some intriguing studies showed that the "yellow flashing" symptom was observed in non-transgenic soybeans and GM soybean only expressed with EPSPS gene after glyphosate application $[7,9,16]$. It is worth noting that damage will easily take place in the actively growing tissues of plants if glyphosate is not sufficiently degraded. The applied glyphosate was accumulated in the meristematic region of TN and GM cottons, and overexpression of EPSPS and GAT genes conferred lower glyphosate residues by glyphosate acetylation in cotton [8]. Similar to the reported results, the "yellow flashing" phenomenon was observed in the terminal leaflets of HJ698, while not in the terminal leaflets of $\mathrm{ZH} 10-6$ when introduction of GAT gene (Fig 1). Therefore, the residual dose of glyphosate after glyphosate acetylation was not enough to cause a toxic effect in ZH10-6. Importantly, the decrease of chlorophyll content is closely related to the "yellow flashing" phenomenon, then affects the photosynthetic process in plant $[10,11]$. However, DEGs associated with function of chlorophyll and photosynthesis pathways were mainly enriched in ZH10-6 at $12 \mathrm{hpt}$ after glyphosate application. Compared to $12 \mathrm{hpt}$, the numbers of DEGs related to photosystem and chlorophyll biosynthesis pathways were obviously increased in two NT soybeans, while slightly increased in HJ698, but significantly decreased in ZH10-6 at 72 hpt (Supplementary Fig. S2 and S3).

Inhibition of plant growth is another typical symptom after glyphosate application [35]. Additionally, synthetic auxin, microtubule and cellulose biosynthesis are critical for plant growth and development process [18]. As was shown in Fig.1, the plant height was significantly inhibited in two NT soybeans at 
$120 \mathrm{hpt}$. Meanwhile, compared to $12 \mathrm{hpt}$, DEGs involved in synthetic auxin and microtubule and cellulose biosynthesis were obviously increased in HJ698 and NT soybeans at $72 \mathrm{hpt}$, but significantly decreased in ZH10-6 at $72 \mathrm{hpt}$. (Supplementary Fig. S6 and S7). These results showed that overexpression of EPSPS gene or/and GAT gene improved the genes expression related to synthetic auxin, and microtubule and cellulose biosynthesis pathways for keeping plant growth. Phenylpropanoid pathway plays an important role in plant growth and development as well as in biotic and abiotic stress responses, and oxidative stress responses [17, 36, 37, 38], and phenylpropanoid pathway also can be found in ZH10, MD12, ZH10 and MD12 (Supplementary Fig. S4 and S5). These results showed that phenylpropanoid pathway, synthetic auxin, microtubule and cellulose biosynthesis were also associated with phenotypic variation among these soybeans. Additionally, the numbers of DEGs involved in shikimate pathway were increased, and their contents of shikimate acid also accumulated rapidly in NT plant [33,34]. Compared to HJ698 and NT soybeans, the number and expression level of DEGs related to shikimate pathway was obviously reduced in $\mathrm{ZH} 10-6$ as the extension of glyphosate post-treatment (Fig. 4). Previous study showed that numbers of DEGs involved in shikimate pathway was not found in NT soybean at $6 \mathrm{hpt}$ after glyphosate treatment, but subsequently increased at $24 \mathrm{hpt}$ and $72 \mathrm{hpt} \mathrm{[18].} \mathrm{Maybe} \mathrm{it} \mathrm{would} \mathrm{take} \mathrm{some} \mathrm{time} \mathrm{for}$ glyphosate uptake before inhibition of shikimate pathway. Surprisingly, the expression trend of $10 \mathrm{DEGs}$ involved in shikimate pathway was completely opposite between ZH10-6 and HJ698 at $12 \mathrm{hpt}$ (Supplementary Fig. S8). These results indicated that the GAT gene might work at the early stage under glyphosate treatment, and subsequently EPSPS gene will be activated when glyphosate uptake.

Taken together, introduction of EPSPS and GAT genes conferred higher tolerance to glyphosate and showed better growth condition, and its comparative transcriptome changes were detected after glyphosate treatment, which will be valuable for further efforts to elucidate molecular mechanisms underlying glyphosate tolerance or glyphosate detoxication.

\section{Abbreviations}

COG: Clusters of Orthologous Groups; DEGs: Differentially expressed genes; EPSPS: 5enolypyruvylshikimate-3-phosphate synthase; FDR: false discovery rate; FPKM: Fragments per Kilobase of transcript per Million fragments mapped; GAT: Glyphosate N-acetyltransferase; GM: Genetically modified; GO: Gene ontology; GOX: Glyphosate oxidoreductase; KEGG: Kyoto Encyclopedia of Genes and Genomes databases; NT: No-transgenic; RNA-Seq: Transcriptome sequencing.

\section{Declarations}

\section{Ethical approval and consent to participate}

Soybeans used in this study were permitted to do scientific research. The experimental research on soybean was complied with Chinese legislation and field studies were in accordance with guidelines of Institute of Crop Science, Chinese Academy of Agricultural Sciences. All the experiments were not involved in any endangered or protected species. 


\section{Consent for publication}

Not applicable.

\section{Conflicts of interest}

The authors declare that they have no conflict of interest.

\section{Acknowledgements}

This work was supported by the National Key R\&D Program of China, Project of Sino-Uruguayan Joint Laboratory (2018YFE0116900); National Transgenic Major Program of China (2016ZX08004001); Young Science Fund Project of Jiangxi (20192ACBL21025) and the Agricultural Science and Technology Innovation Program (ASTIP) of Chinese Academy of Agricultural Sciences.

\section{Availability of data and materials}

Data pertaining to the study have been included in this manuscript and supplementary materials.

\section{Author contributions}

B.G., Y.G., H.H., and L.J.Q. conceived and designed the experiments. B.G., H.H., and Y.G. performed the experiments. B.G. and Y.G. analyzed the data. B.G., Y.G. and L.J.Q. wrote and revised the manuscript.

\section{References}

1. Hartman GL, West ED, Herman TK (2011) Crops that feed the world 2. Soybean-worldwide production, use, and constraints caused by pathogens and pests. Food Secur 3(1): 5-17

2. Hollander H, Amrhein N (1980) The site of the inhibition of the shikimate pathway by glyphosate: I. Inhibition by glyphosate of phenylpropanoid synthesis in buckwheat (Fagopyrum esculentum Moench). Plant Physiol 66(5): 823-829

3. Steinrucken HC, Amrhein, N (1980) The herbicide glyphosate is a potent inhibitor of 5-enolpyruvylshikimic acid-3-phosphate synthase. Biochem Biophys Res Commun 94(4): 1207-1212

4. Padgette SR, Taylor NB, Nida DL, Bailey MR, MacDonald J, Holden LR, Fuchs RL (1996) The composition of glyphosate-tolerant soybean seeds is equivalent to that of conventional soybeans. $J$ Nutr 126: 702-716

5. Meilan R, Han KH, Ma C, DiFazio SP, Eaton J, Hoien EA, Stanton BJ, Crockett RP, Taylor ML, James RR, Skinner JS, Jouanin L, Pilate G, Strauss SH (2002) The CP4 transgene provides high levels of tolerance to Roundup ${ }^{\circledR}$ herbicide in field-grown hybrid poplars. Can J Res 32(6): 967-976

6. Tian YS, Xu J, Xiong AS, Zhao W, Fu XY, Peng RH, Yao QH (2011) Improvement of glyphosate resistance through concurrent mutations in three amino acids of the ochrobactrum 5enopyruvylshikimate-3-phosphate synthase. Appl Environ Microbiol 77(23): 8409-8414 
7. Pline WA, Wilcut JW, Duke SO, Edmisten KL, Wells R (2002) Tolerance and accumulation of shikimic acid in response to glyphosate applications in glyphosate-resistant and non-resistant cotton (Gossypium hirsutum L). J Agric Food Che. 50(3): 506-512

8. Liang CZ, Sun B, Meng ZG, Wang Y, Sun GQ, Zhu T, Lu W, Zhang W, Malik WQ, Lin M, Zhang R, Guo SD (2017) Co-expression of GR79 EPSPS and GAT yields herbicede-resistant cotton with low glyphosate residues. Plant biotechnol J 15(12): 1622-1629

9. Zobiole LHS, Kremer RJ, Oliveira RS, Constantin J (2012) Glyphosate effects on photosynthesis, nutrient accumulation, and nodulation in glyphosate-resistant soybean. J Plant Nutr Soil Sci 175(2): 319-330

10. Campbell WF, Evans JO, Reed SC (1997) Effect of glyphosate on chloroplast ultrastructure of quackgrass mesophyll cells. Weed Sci 24(1): 22-25

11. Reddy KN, Rimando AM, Duke SO (2004) Aminomethyl phosphonic acid, a metabolite of glyphosate, causes injury in glyphosate-treated, glyphosate-resistant soybean. J Agric Food Chem 52(16): 51395143

12. Castle LA, Daniel LS, Gorton R, Patten PA, Chen YH, Bertain S, Cho HJ, Duck N, Wong J, Liu DL, Lassner MW (2004) Discovery and directed evolution of a glyphosate tolerance gene. Science 304 (5674): 1151-1154

13. Siehl DL, Castle LA, Gorton R, Chen YH, Bertain S, Cho HJ, Keenan R, Liu DL, Lassner MW (2005) Evolution of a microbial acetyltransferase for modification of glyphosate: a novel tolerance strategy. Pest Manag Sci 61(3): 235-240

14. Pedotti M, Rosini E, Molla G, Moschetti T, Savino C, Vallone B, Pollegioni L (2009) Glyphosate resistance by engineering the flavoenzyme glycine oxidase. J Biol Chem. 284(52): 36415-36423

15. Dun BQ, Wang XJ, Lu W, Chen M, Zhang W, Ping SZ, Wang ZX, Zhang BM, Lin M (2014) Development of highly glyphosate-tolerant tobacco by coexpression of glyphosate acetyltransferase gat and EPSPS G2-aroA Crop J 2(2), 164-169

16. Guo BF, Guo Y, Hong HL, Jin LG, Zhang LJ, Chang RZ, Lu W, Lin M, Qiu LJ (2015) Co-expression of G2-EPSPS and glyphosate acetyltransferase GAT genes conferring highly glyphosate tolerance in soybean. Front Plant Sci 6: 847

17. Zhu J, Patzoldt WL, Shealy RT, Vodkin LO, Clough SJ, Tranel PJ (2008) Transcriptome response to glyphosate in sensitive and resistant soybean. J Agric Food Chem 56(15): 6355-6363

18. Jiang LX, Jin LG, Guo Y, Tao B, Qiu LJ (2013) Glyphosate effect on the gene expression of the apical bud in soybean (Glycine max). Biochem Bioph Res Commun 437(4): 544-549

19. Florea L, Song L, Salzberg SL (2013) Thousands of exon skipping events differentate among splicing patterns in sixteen human tissues. F1000 Res 2: 188

20. Leng N, Dawson JA, Thomson JA, Ruotti V, Rissman Al, Smits BMG, Haag J, Gould MN, Stewart R (2013) EBSeq: an empirical Bayes hierarchica Imodel for inference in RNA-seq experiments. Bioinformatics 29(8): 1035-1043 
21. Kenneth JL, Schmittgen TD (2001) A analysis of relative gene expression data using Real-Time quantitativa PCR and the $2^{-\Delta \Delta C T}$ Method 25(4) 402-408

22. Deng YY, Li JQ, Wu SF, Zhu YP, Chen YW, He FC (2006) Integrated nr database in protein annotation system and its localization. Comput Eng 32: 71-72

23. Ashburner M, Ball CA, Blake JA, Botstein D, Butler H, Cherry JM, Davis AP, Dolinski K, Dwight SS, Eppig JT, Harris MA, Hill DP, Lssel-Tarver L, Kasarskis A, Lewis S, Matese JC, Richardson JE, Ringwald M, Rubin GM, Sherlock G (2000) Geneontology: tool for the unification of biology. Nat Genet 25(1): 25-29

24. Tatusov RL, Galperin MY, Natale DA, Koonin EV (2000) The COG database: a tool for genome-scale analysis of protein functions and evolution. Nucleic Acids Res 28(1): 33-36

25. Apweiler R, Ball CA, Wu CH, Baker WC, Boeckmann B, Ferro S (2004) Uniprot: the universal protein knowledgebase. Nucleic Acids Res 32(1): D115-D119

26. Kanehisa M, Goto S, Kawashima S, Okuno Y, Hattori M (2004) The KEGG resources for deciphering the genome. Nucleic Acids Res 32(1): D277-280

27. Conesa A, Götz S, García-Gómez JM, Terol J, Talón M, Robles M (2005) Blast2 GO: auniversal tool for annotation,visualization and analysis in functional genomics research. Bioinformatics 21(18): 36743676

28. Kim D, Pertea G, Trapnell C, Pimentel H, Kelley R, Salzberg SL (2013) TopHat2: accurate alignment of transcriptomes in the presence of insertions, deletions and gene fusions. Genome Biol 14: R36

29. Schulze SK, Kanwar R, Golzenleuchter M, Therneau TM, Beutler AS (2012) SERE: Single-parameter quality control and sample comparison for RNA-Seq. BMC Genomics 13(1): 524

30. Monquero PA, Christoffoleti PJ, Osuna MD, Deprado, RA (2004) Absorption, translocation and metabolism of glyphosate by plants tolerant and susceptible to this herbicide. Planta Daninha 22(3): 445-451

31. Burton JD, Balke NE (1988) Glyphosate uptake by suspension-cultured potato (Solanum tuberosum and S. brevidens) Cells. Weed Sci 36(2): 146-153

32. Hernandez A, Garcia-Plazaola JI, Becerril JM (1999) Glyphosate effects on phenolic metabolism of nodulated soybean (Glycine max merr). J Agric Food Chem 47(7): 2920-2925

33. Mueller TC, Massey JH, Hayes RM, Chris L, Stewart CN (2003) Shikimate accumulates in both glyphosate-sensitive and glyphosate-resistant horseweed (Conyzacanadensis Cronq.). J Agric Food Chem 51(3): 680-684

34. Henry WB, Shaner DL, West MS (2007) Shikimate accumulation in sunflower, wheat, and prosomillet after glyphosate application. Weed Sci 55(1): 1-5

35. Dixon RA, Lamb CJ, Masoud S, Sewalt VJ, Paiva NL (1996) Metabolic engineering: prospects for crop improvement through the genetic manipulation of phenylpropanoid biosynthesis and defense responses-a review. Gene 179(1): 61-71 
36. Naoumkina MA, Zhao Q, Gallego-Giraldo L, Dai X, Zhao PX, Dixon RA (2010) Genome-wide analysis of phenylpropanoid defence pathways. Mol Plant Pathol 11(6): 829-46

37. Yamaguchi M, Valliyodan B, Zhang J, Lenoble ME, Yu O, Rogers EE, Nguyen HT, Sharp RE (2010) Regulation of growth response to water stress in the soybean primary root. I. Proteomic analysis reveals region-specific regulation of phenylpropanoid metabolism and control of free iron in the elongation zone. Plant Cell Environ 33(2): 223-43

38. Tohge T, Watanabe M, Hoefgen R, Fernie AR (2013) The evolution of phenylpropanoid metabolism in the green lineage. Crit Rev Biochem Mol Biol 48(2): 123-52

39. Gazziero DLP, Adegas F, Voll E (2008) Glifosate e Soja Transgênica. Circular Técnica 60. Londrina: Embrapa Soja, p4.

40. Hiroshi M, Natalia D (2012) The shikimate pathway and aromatic amino acid biosynthesis in plants. Annu Rev Plant Biol 63: 73-105

\section{Figures}




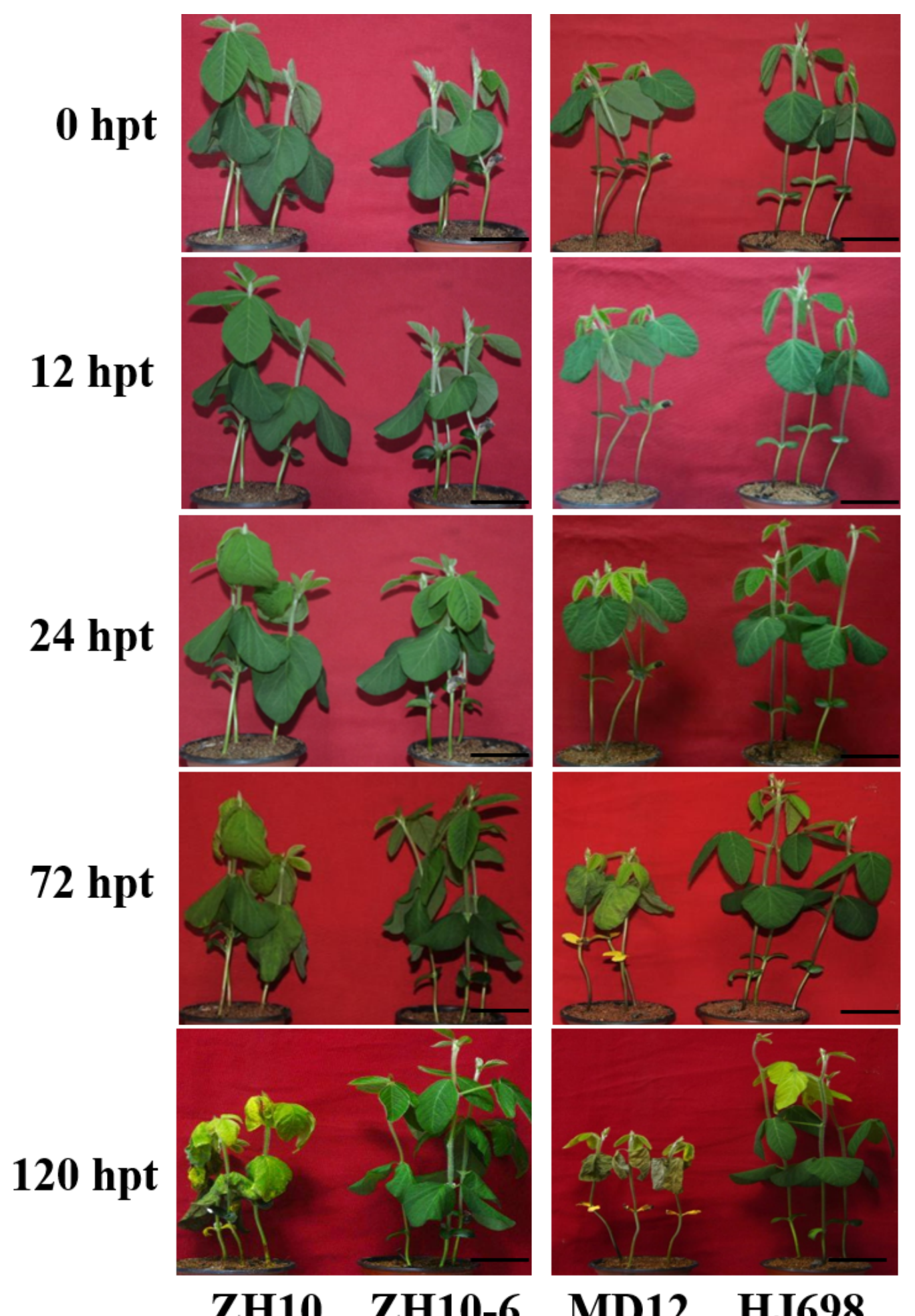

\section{ZH10 ZH10-6 MD12 HJ698}

\section{Figure 1}

Glyphosate tolerance analysis in four soybean genotypes . Seedlings of ZH10, ZH10-6, MD12, HJ698 were spraying with Roundup ${ }^{\circledR}$ at rate of $0.18 \mathrm{~g}$ a.e. $/ \mathrm{m} 2$ when their first trifoliolate leaves were fully expanded. Then their growth condition was analyzed in these soybean genotypes at $0,12,24,72$, and 120 hpt. Black bars $=5 \mathrm{~cm}$. 
$\mathbf{a}$

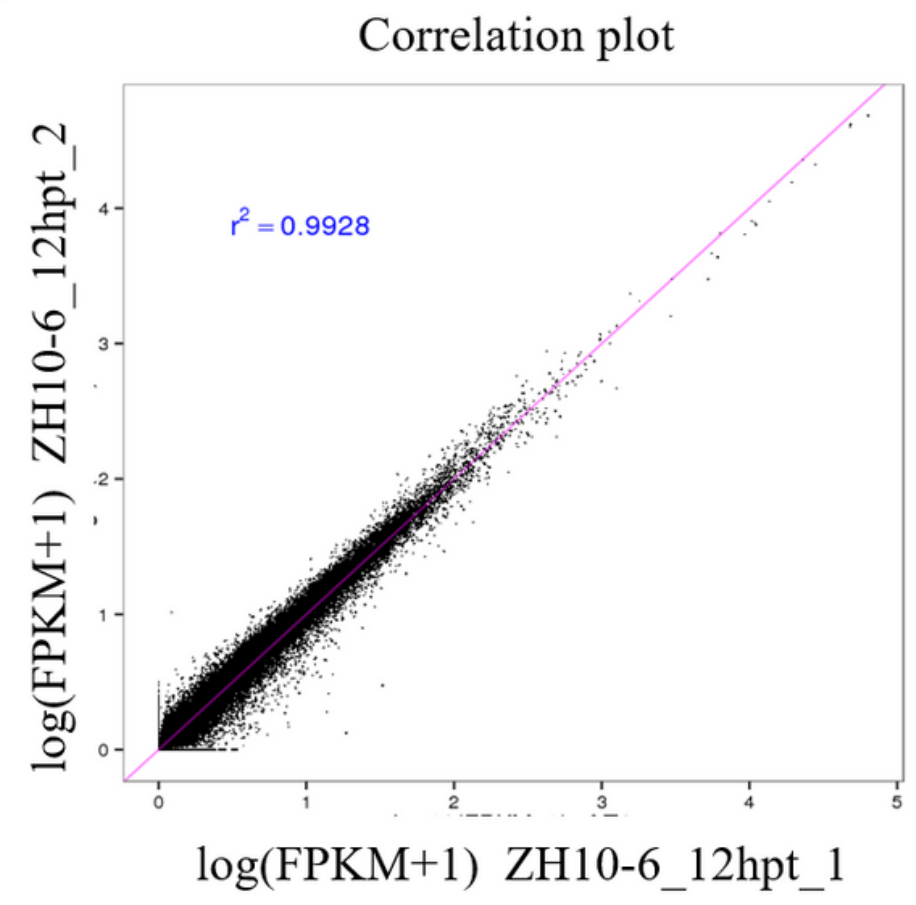

b

Correlation plot

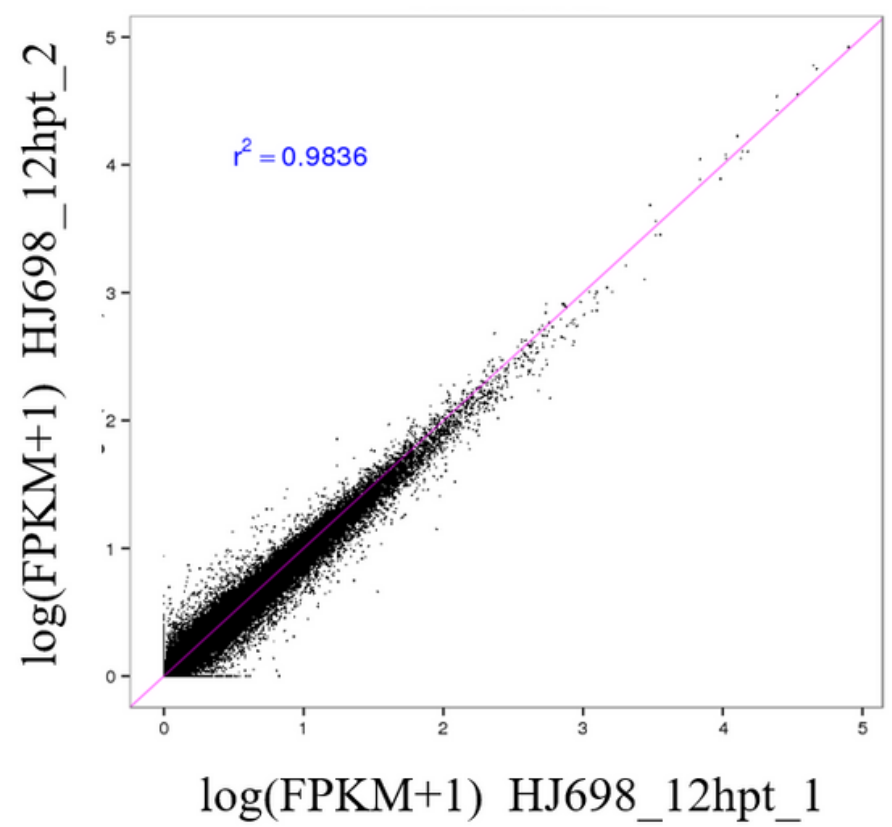

Figure 2

Correlation analysis of replicated samples of ZH10-6_12hpt (a) and HJ698_12hpt (b). 
a

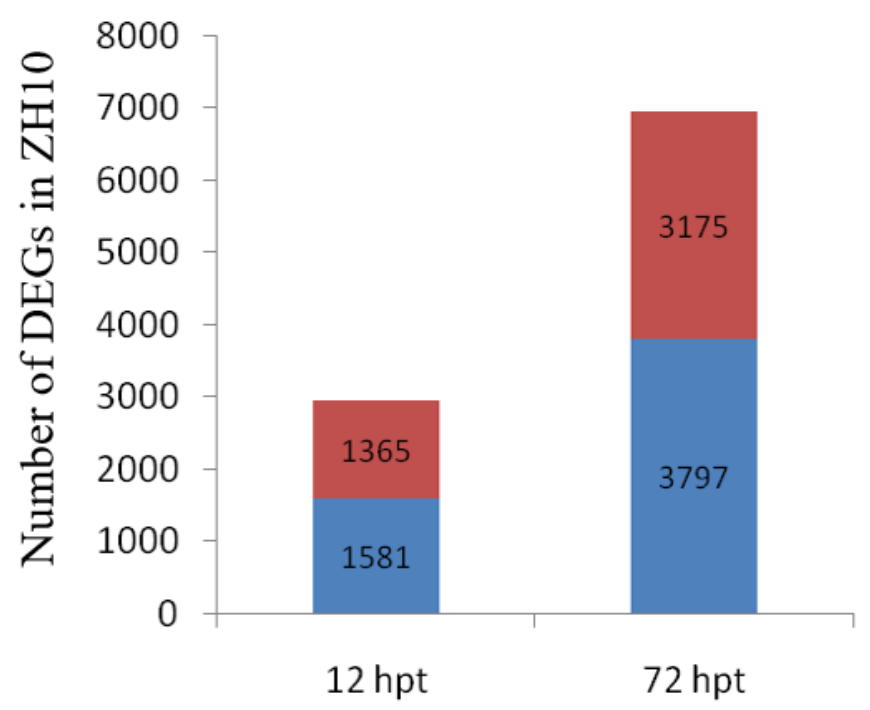

C

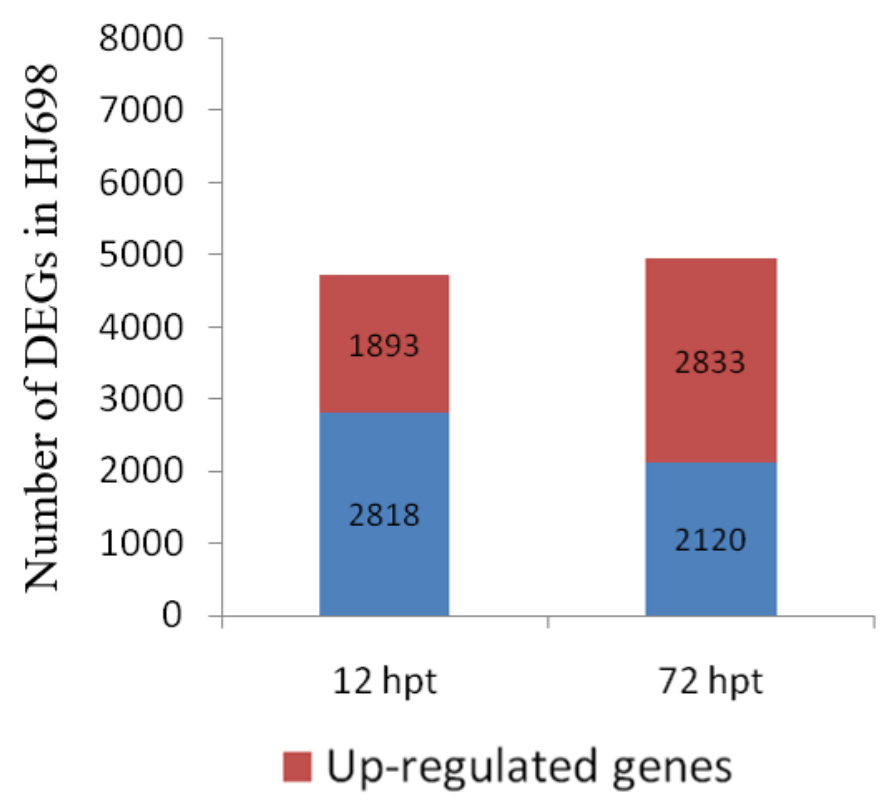

b

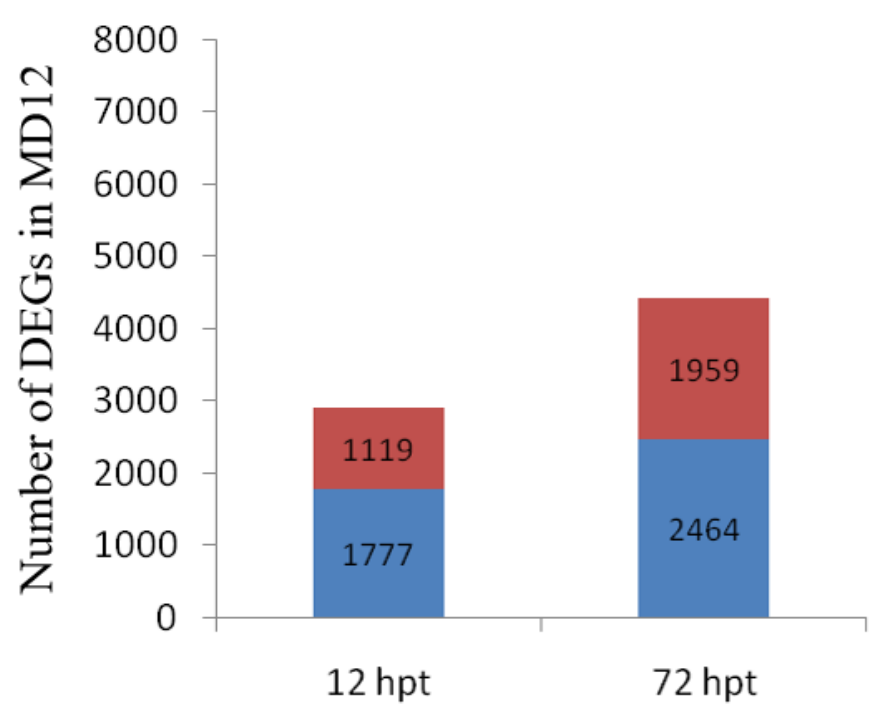

d

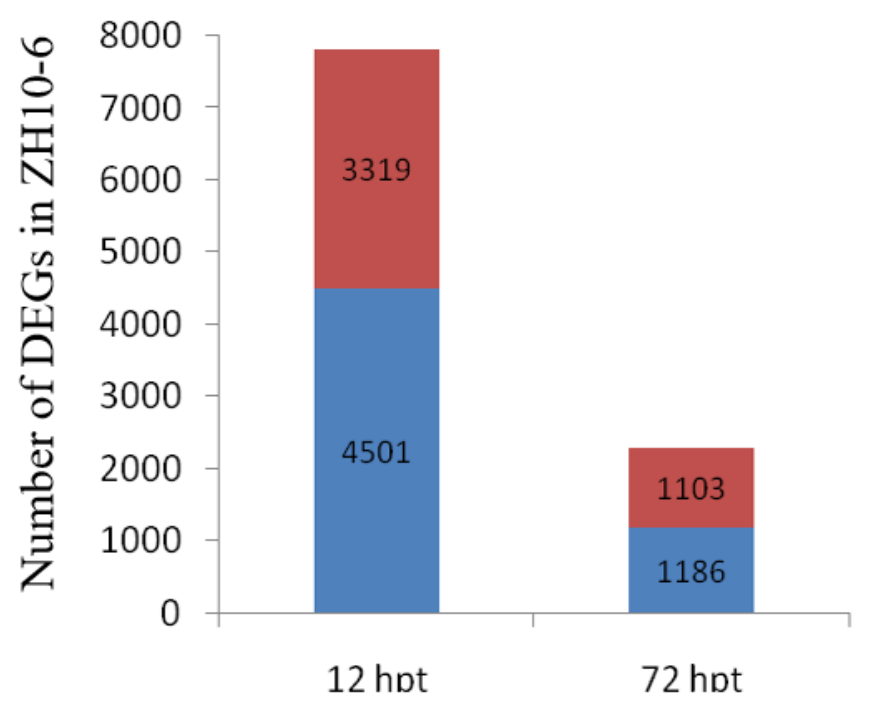

Down-regulated genes

\section{Figure 3}

Diagram showing the number of DEGs response to glyphosate treatment in four soybean genotypes ZH10 (a), ZH10-6 (b), MD12 (c), and HJ698 (d). 


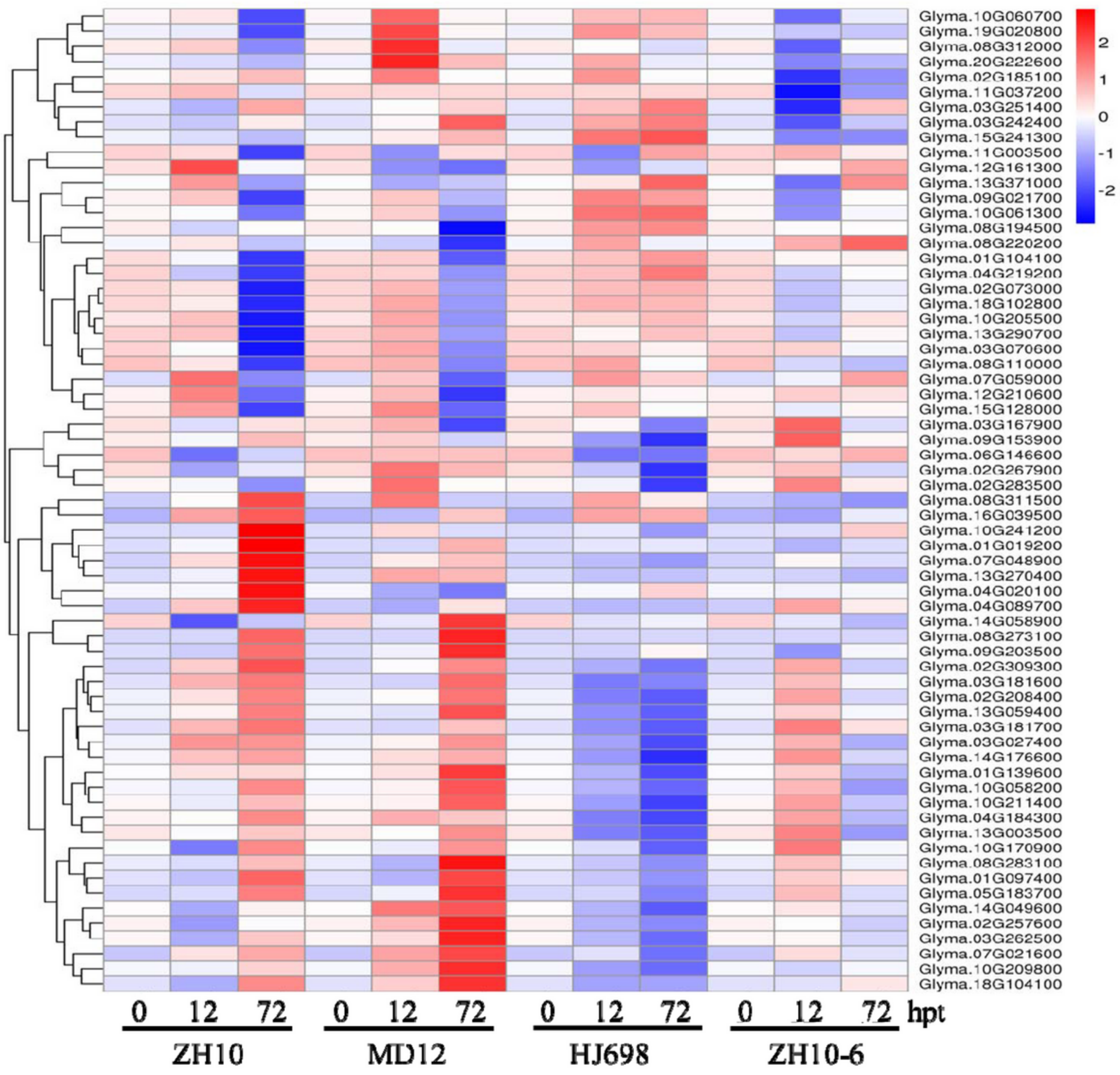

Figure 4

Heatmap analysis of FPKM values of DEGs involved in shikimate pathway in four soybeans.Genes involved in shikimate pathway was selected by homolog identification. Heatmap analysis was conducted by R Project. Blue color indicated low expression level while red color indicated high expression level. 


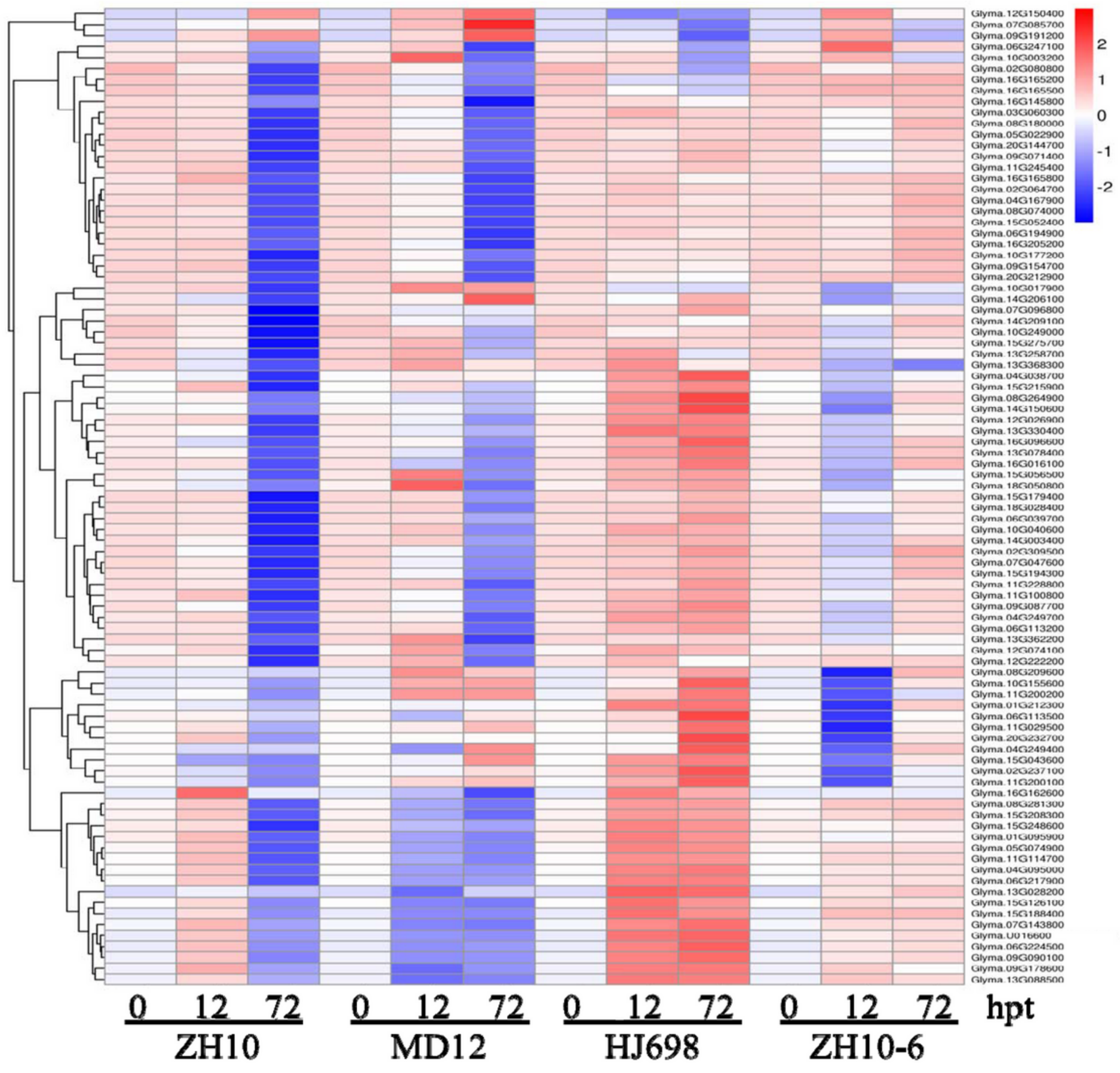

Figure 5

Heatmap analysis of FPKM values of DEGs involved in photosystem and chlorophyll biosynthesis in four soybeans. Genes involved in shikimate pathway was selected by homolog identification. Heatmap analysis was conducted by R Project. Blue color indicated low expression level while red color indicated high expression level.

\section{Supplementary Files}

This is a list of supplementary files associated with this preprint. Click to download. 
- SupplementalFigureS1.tif

- SupplementalFigures2.tif

- SupplementalFigureS3.tif

- SupplementalFigureS4.tif

- SupplementalFigureS5.tif

- SupplementalFigureS6.tif

- SupplementalFigureS7.tif

- SupplementalFigureS8.tif

- SupplementalTableS1.xIsx

- SupplementalTableS2.xlsx

- SupplementalTableS3.xIsx

- SupplementalTableS4.xIsx 